\title{
Reinterpretation of the mechanical reinforcement of polymer nanocomposites reinforced with cellulose nanorods
}

\author{
Janak Sapkota $\mathbb{D}^{1}{ }^{1}$ Julio Cesar Martinez Garcia, ${ }^{2}$ Marco Lattuada ${ }^{2}$ \\ ${ }^{1}$ Chair of Polymer Processing, Department of Polymer Engineering and Science, Montanuniversitaet Leoben, Leoben 8700 , Austria \\ Department of Chemistry, University of Fribourg, Fribourg 1700, Switzerland \\ Correspondence to: J. Sapkota (E-mail: janak.sapkota@unileoben.ac.at) and J. C. Martinez Garcia (E-mail: jmartinezgarcia@gmail.com)
}

\begin{abstract}
The mechanical reinforcement of nanocomposites containing nanorods-like fillers such as cellulose nanocrystals (CNCs) is often interpreted by adapting the classical parallel-series model, assuming a simple hyperbolic dependence between the percolation threshold and aspect ratio. However, such assumptions are valid only for nanorods with high aspect ratio and often are misinterpreting the reinforcement obtained at low volume fraction of filler loading. To elucidate this intriguing scenario, we proposed a new approach and validated it by compiling and reinterpreting some of available literature that represent the experimental reinforcement with CNCs. Our approach showed better accuracy, specifically for the cases of CNC nanorods with lower aspect ratio. We conclude that this route permits a more realistic evaluation of the mechanical reinforcement, where a physical parameter accounting the polymer filler association is introduced. () 2017 Wiley Periodicals, Inc. J. Appl. Polym. Sci. 2017, 134, 45254.
\end{abstract}

KEYWORDS: composites; mechanical properties; nanocrystals; nanotubes; structure-property relations; theory and modeling

\section{INTRODUCTION}

Cellulose nanocrystals (CNCs) are typically rigid rod-shaped monocrystalline cellulose with varying aspect ratio depending on the origin and methods of extraction. ${ }^{1}$ Besides the ecofriendliness, sustainable, and cost competitiveness, CNCs have enriched surface active groups and can be easily incorporated in commercial polymers to obtain high-performance nanocomposites. ${ }^{1-6}$ A precise interpretation of the physical properties such as mechanical reinforcement of these nanocomposites is crucial for fundamental understanding and designing and producing novel nanocomposite materials with optimized properties.

The formation of the inter-particle/interconnected clusters or networks within a polymer matrix, formulated in-terms of geometric proximity, is one effective manner to massively enhance mechanical reinforcement of nanocomposites. ${ }^{7,8}$ Above a certain critical volume fraction (referred to as percolation threshold), rod-shaped fillers, such as carbon nanotubes (CNT) or CNCs, interconnect to create a network inside the matrix, which usually leads to a step-wise increase in some physical properties. Examples of these properties include mechanical resistance and electric conductivity, if the fillers are electrically conductive. Several works, both based on theories and simulations, have revealed that the percolation threshold depends upon the distribution of shape, size, and orientation of the nanofillers. ${ }^{9-16}$ Meanwhile, several experimental research efforts have been devoted to understanding the formulation of the composites and the influence of processing conditions on the interconnectivity and the reinforcing behavior. ${ }^{2,3,17-24}$ However, in all of these studies, irrespective of the polymer matrices, a homogenous dispersion of CNCs in the nanocomposite is assumed and hence the formation of $\mathrm{CNC}-\mathrm{CNC}$ interconnectivity via local clusters or networks is idealized, giving rises to a significant increase of the storage modulus of the nanocomposites, referred as mechanical reinforcement. The influence of the different polymer matrix on the mechanical reinforcement of CNC nanocomposites has so far been neglected by most of the available approaches. Therefore, a more realistic understanding and precise estimation of the mechanical reinforcement is missing.

The mechanical properties of nanocomposites of CNCs with soft polymers (above $\left.T_{g}\right)^{2,3,17-24}$ has been analyzed based on several theoretical models that build on the approach developed by Ouali et al., ${ }^{25}$ who had improved the classical parallel-series model developed by Takayanagi et al. ${ }^{26}$ The improvement was carried out by making the assumption that a composite material is made not only of polymer and filler, but also of a third phase, the percolating network, which appears only above a certain threshold. The percolation assumption (i.e., power law 
behavior around the percolation threshold) describes how the effective fraction of nanorods belonging to the percolating network contributes to the mechanical reinforcement. According to the model by Ouali-Takayanagi, the tensile storage modulus $E^{\prime \prime}$ of $\mathrm{CNC}$ nanocomposites can be expressed as a function of the volume fraction of CNCs $X_{n}$ the tensile modulus $E_{r}$ of the rigid filler phase, and the modulus of the matrix polymer $\left(E_{s}\right)$ by:

$$
\begin{gathered}
E^{\prime}=\frac{\left(1-2 \phi+\phi X_{r}\right) E_{s} E_{r}+\left(1-X_{r}\right) \phi E_{r}^{2}}{\left(1-X_{r}\right) E_{r}+\left(X_{r}-\phi\right) E_{s}} \\
\phi=\left\{\begin{array}{cc}
0 & : X_{r}<\psi_{c} \\
X_{r}\left(\frac{X_{r}-\psi_{c}}{1-\psi_{c}}\right)^{b} & : X_{r}>\psi_{c}
\end{array}\right\}
\end{gathered}
$$

being $(\phi)$ the effective volume fraction of CNCs that contribute to the formation of the percolation network. The percolation exponent (b) in eq. (2) is assumed to be equal to 0.4 as predicted by Stauffer and Aharony ${ }^{27}$ and de Gennes ${ }^{28}$ for three-dimensional systems.

Several models that are capable to predict the percolation threshold in composite materials containing fibers, such as CNTs and CNCs have been reported. ${ }^{11-13,29-36}$ However, for nanocomposite with low aspect ratio, such as those containing the CNCs derived from cotton (aspect ratio, $\gamma=L / D \approx 12$ ), most of the experimental elastic storage moduli data reported so far have been evaluated and interpreted by adapting Ouali-Takayagi model using an inverse aspect ratio scaling (hyperbolic relationship) to predict the percolation threshold. This approach is known to be accurate only in the case of fillers with a sufficiently high aspect ratio $(\gamma>60)$, but diverges especially at lower filler volume fraction with experimental results when CNCs of low aspect ratio $(\gamma<10)$ are used. However, one of the great practical relevance of CNCs is that their availability and (projected) costs are highly favoring CNCs with lower aspect ratio. ${ }^{1,6}$ To understand this divergence, one has to look at the assumptions behind the conventional approach which shows three crucial points that seem to be ignored: (a) The assumption of hyperbolic dependence is valid only in the limit of infinitely slender rods (i.e., with higher aspect ratio), ${ }^{37,38}$ (b) The established evidences (both from experiments and from simulations) of the matrix-filler interface for the reinforcement effect is omitted, (c) Percolation threshold is evaluated as an exclusive function of the aspect ratio, where other possible relevant physical variables are omitted. In order to elucidate these unclear points, a more consistent realistic approach is needed.

Recently Schilling et al. ${ }^{39}$ proposed a new percolation theory that is capable of accurately predicting the percolation threshold for rod-like particles, from spheres to needles, and validated against Monte Carlo simulations. This novel approach expresses the percolation threshold as a function of two variables: the aspect ratio of the rods and the inter-particle connectivity $(\alpha)$, which is related to the thickness of a shell surrounding the rods that participates in the formation of the percolating network. However, the thickness of a shell surrounding the rods could possibly be influenced by the nature/type of polymer matrices in which nanorods are dispersed, likely due to polymer-filler interactions, and this has been overlooked.
For a better understanding of the mechanical reinforcement it is very important to evaluate the role of the filler-filler and fillerpolymer matrix interaction in term of both the filler aspect ratio and the filler-polymer association effect. ${ }^{40-43}$ The use of an equation capable of correctly predicting the percolation threshold even in the case of low aspect ratio filler $(\gamma<10)$ that would enable to investigate systematically the mechanical reinforcement is missing, and this could have a great implications in understanding and developing new composites. ${ }^{34,35}$ On the other hand, in comparison with the previous percolation theories, the approach by Schilling et al. $^{39}$ is one of the models capable of precisely predicting the percolation threshold from spheres to needles by introducing a new parameter $(\alpha)$ representing a dimensionless measure of the particle connectivity, which could be directly correlated with the fillerpolymer association. Experimental evidences based on nuclear magnetic resonance spectroscopy have indicated the presence of a glassy layer around the unmodified filler particles ${ }^{44}$ and it is also claimed that changes in the matrix properties (e.g., molecular weight) close to the filler surface influences strongly the reinforcement. ${ }^{45}$ In addition to that, recently Gravilov et al. ${ }^{46}$ have used a large-scale dissipative dynamics simulation to study the structural changes in unfilled and filled elastomers during uniaxial deformation demonstrating that the properties of the polymer chains are strongly modified by the presence of solid particles, being the reason of the abrupt increase of the composite storage modulus.

To elucidate the gaps of conventional approach, this rapid communication proposes a new approach to interpret the mechanical reinforcement in polymer nanocomposite reinforced with nanorods, such as CNCs. By using our method, we have evaluated and compared our findings with the experimental measurements (obtained directly from the literature) the dependence of the elastic storage moduli for CNCs-reinforced polymeric composites for four different set of polymer matrices. For these four sets, two subsets are considered where CNCs from different sources and hence their aspect ratios are different. Our method allows us to determine the connectivity parameter for every polymer nancomposites, which is an important factor we should consider for interpreting the mechanical reinforcement in polymer nanocomposites effectively.

\section{OUR APPROACH}

Our method is based on the adaptation of eq. (1) with an appropriate approach capable to compute the percolation threshold $\psi_{c}$ of eq. (2) in a very consistent way. As a part of our method, we have selected the percolation model introduced by Schilling et al. ${ }^{39}$ In this model, the particles are considered as spherocylinders of length $L$ and diameter $D$ as shown schematically in Figure 1, with overlapping contact shells diameter $\lambda>D$. The particles are connected if their shells overlap, and clusters are defined by contiguous pairwise connections. The percolation threshold is then expressed as function of the aspect ratio $\gamma=L / D$ and the connectivity variable $\alpha=(\lambda / D-1)$ by:

$$
\psi_{c}=\frac{2(1+\xi)-2\left(1+\frac{\xi}{2}\right)^{\frac{1}{2}}}{3\left(1+\frac{2}{3} \xi\right)}
$$

where 


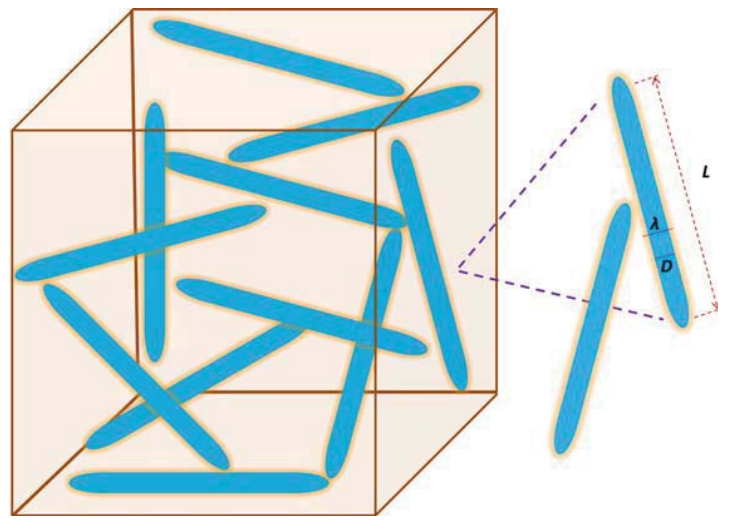

Figure 1. Schematic representation of a percolating clusters of CNCs (represented as spherocylinder with length $L$ and diameter $D$ ) in nanocomposites. CNCs are supposed to be in contact with each other with overlapping contact shells of diameter $\lambda$ (orange shade). [Color figure can be viewed at wileyonlinelibrary.com]

$$
\xi(\gamma, \alpha)=\frac{\left(1+\frac{2}{3}\left(\frac{1}{\gamma}\right)\right) \frac{1}{\gamma}}{\frac{8}{3 \gamma^{2}}\left((1+\alpha)^{3}-1\right)+\frac{4}{\gamma}\left((1+\alpha)^{2}-1\right)+\alpha}
$$

\section{RESULTS AND DISCUSSION}

The combination of eqs. (1)-(4) offers us the possibility to extract the connectivity parameter $\alpha$, directly from the tensile storage modulus $E^{\prime}$ data fitting using experimentally determined parameters. Figure 1 shows the dependence of the elastic storage moduli for CNCs-reinforced polymeric composites for different polymers, viz ethyleneoxide-epichlorohydrin copolymer (EOEPI), poly(vinly alcohol) (PVA), epoxy, and poly(vinly acetate) (PVAc). The black squares and red circles represents the reported experimental data for the case of nanocomposites with tunicate CNCs (tCNCs, having high aspect ratio, ca. $\gamma=84$ ) and nanocomposites with cotton CNCs (cCNCs, having low aspect ratio, ca. $\gamma=11$ ) respectively. The dotted lines (black for tCNCs and red for cCNCs) show the fitting of the experimentally reported values using Ouali-Takayanagi model with the hyperbolic assumption as estimate of the percolation threshold, whereas the solid line shows the analogous fitting with our new method (The detailed fitting parameters are presented in Supporting Information Table S1). Figure 2 clearly shows that for the fraction of CNCs $X_{r} \gg \psi_{c}$ the role of connectivity parameter will be insignificant to the change of the polymer matrix. It can be justified because the elastic storage moduli predicted by Ouali-Takayanagi model will converge to $E_{r}$ independently of the percolation threshold assumption. However the situation is clearly different for the fraction of CNCs at $X_{r} \approx \psi_{c}$.

For tCNCs (CNCs with high aspect ratio), in the vicinity of $\psi_{c}$, there is just a slight discrepancy between both approaches. This small difference can be justified because the percolation threshold predicted by Schilling's model differs little from the percolation predicted by hyperbolic scaling assumption for rods with large aspect ratios. However,
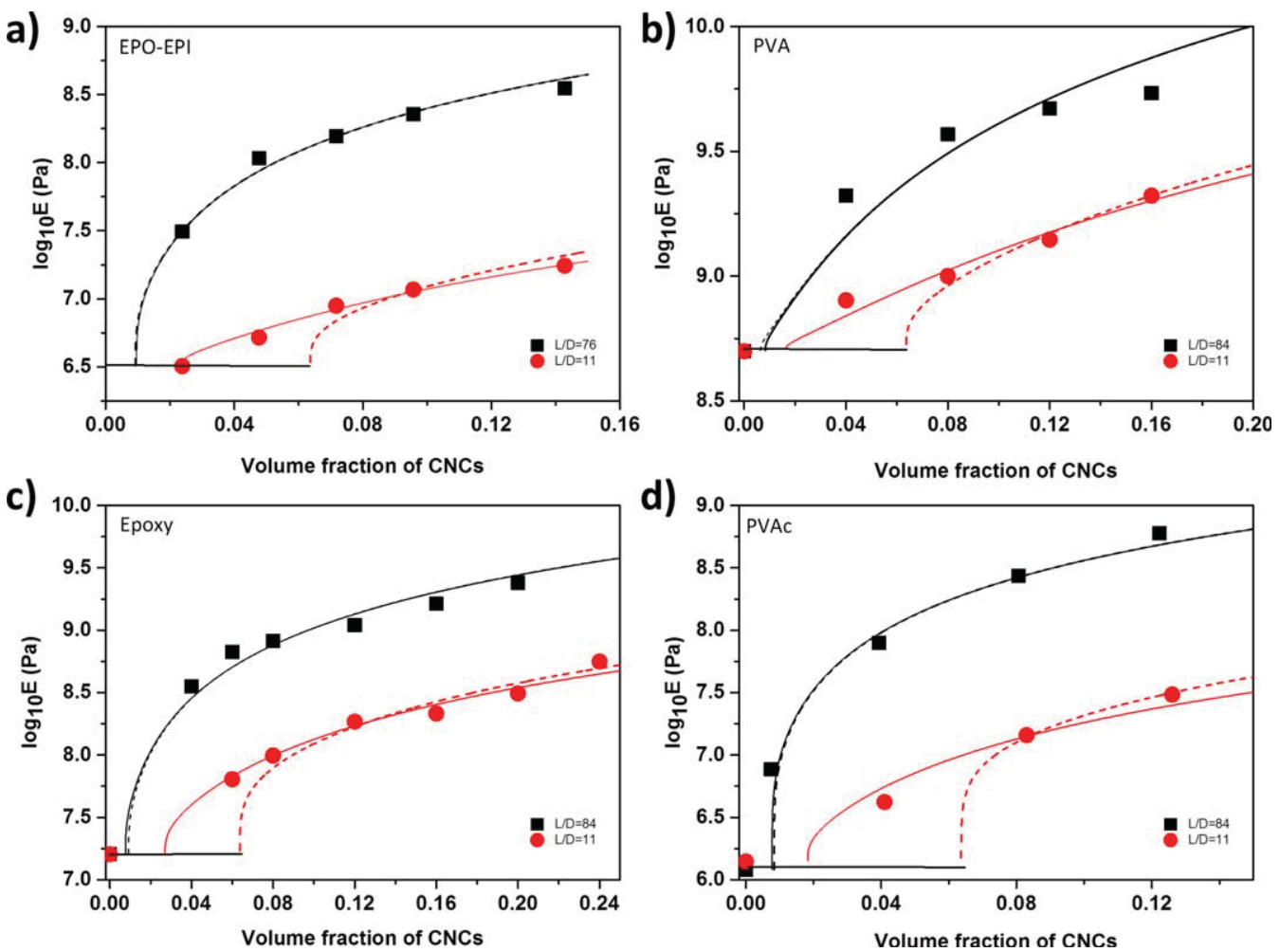

Figure 2. Elastic storage moduli for CNCs reinforced polymers: (a) EO-EPI (adapted from Ref. 1), (b) PVA (adapted from Ref. 22), (c) Epoxy (adapted from Ref. 47), and (d) PVAc (adapted from Ref. 3). The red lines correspond to the nanocomposite with CNCs derived from cotton (cCNCs, low aspect ratio), and black line corresponds to the nancomposites with CNCs derived from tunicate (tCNCs, high aspect ratio). [Color figure can be viewed at wileyonlinelibrary.com] 

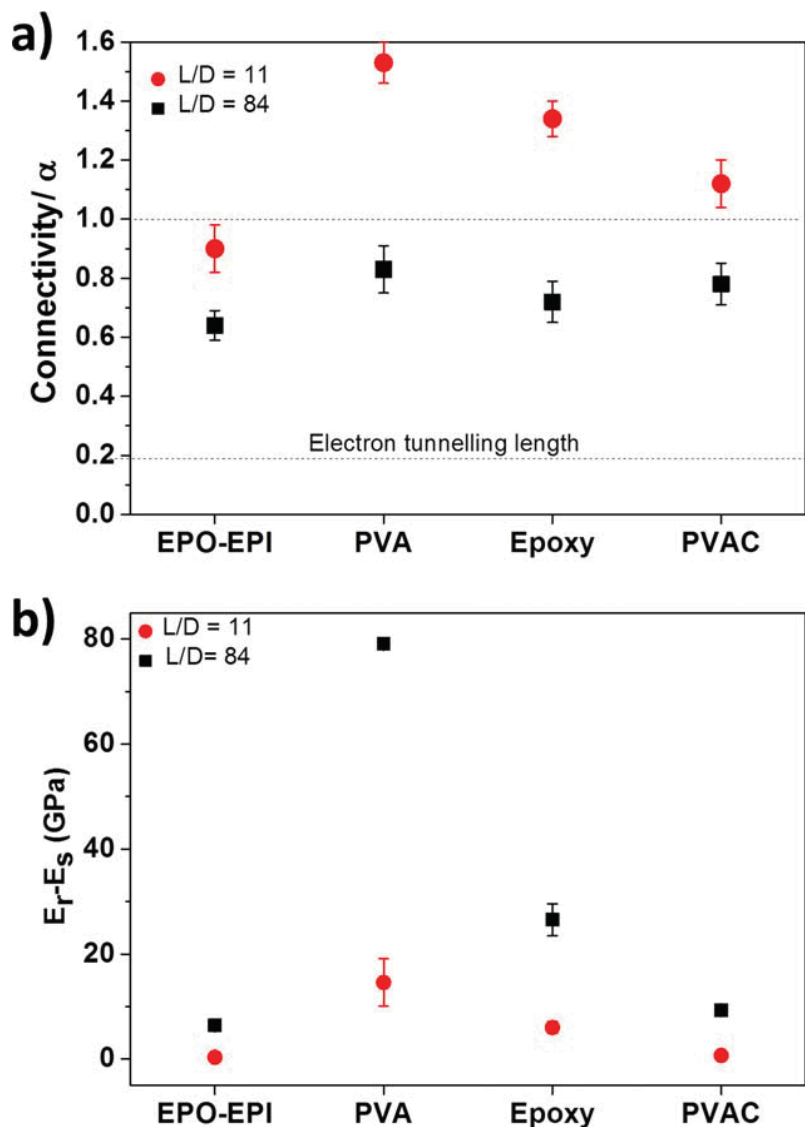

Figure 3. (a) Extracted connectivity parameters related to the thickness of a shell surrounding the nanorods of different aspect ratios that participates to the formation of the percolating network. The dotted line $(\alpha=0.2)$ is ascribed to the connectivity parameter used by Schilling et al. ${ }^{39}$ for their model validation. (b) Comparisons of the reinforcement as a function of the type of polymer for mechanical percolation. EO-EPI, PVA, epoxy, and PVAc. [Color figure can be viewed at wileyonlinelibrary.com]

for $\mathrm{CCNCs}$, in the vicinity of $\psi_{o}$ the predictions of both approaches are considerably different. The results obtained by using the hyperbolic adaptation (red dotted lines) are acceptable only in the case of nanocomposites with high volume fraction of cCNCs. The conventional approach seems to ignore the reinforcement $\left(E>E_{s}\right)$ at lower volume fraction of $\mathrm{cCNCs}$, i.e., below the percolation threshold limit. On the other hand, for each polymer system, the $\psi_{c}$ values predicted by our updated approach are considerably smaller than those predicted by the conventional hyperbolic scaling and matched better with the experimental results. The fitting suggests that for the case of $\mathrm{cCNCs}$, the polymer matrix can influence significantly the percolation network formation, and this fact is often ignored, even though the filler geometry and distribution are considered the dominant factors to determine the percolation threshold. Nevertheless, the host polymer matrix can impact the dispersion and hence the clusters/network geometry of CNCs. For the low aspect ratio CNC, it suggests that the inter-particle connectivity distance of the particles is much larger for the selected behavior.

Figure 3(a) shows the $(\alpha)$ values determined from our method. It is clearly visible that for each case the surface shell size of the filler-polymer will be smaller for rods with larger aspect ratio than for those with small aspect ratio. This is true as particles with higher aspect ratio are more easily kinetic arrested in comparison with the lower aspect ratio particles, causing kinetic jamming in the nanocomposites. ${ }^{48}$ This is also reflected in the connectivity parameter values of all the systems evaluated in the present work. For the nanocomposites with tCNCs, the connectivity parameter changed (from 0.65 for EO-EPI to 0.85 for epoxy, see Supporting Information Table S2) whereas for the same nanocomposites when cCNCs are used instead of tCNCs, the parameter changes dramatically (from 0.9 for EO-EPI to 1.53 for PVA). These results suggest that the polymer matrix has significant influence in co-assembly of the cCNCs and hence the cluster/network formation. This is also in accordance with recent study ${ }^{49}$ which shows that the interphase thickness depends on the CNC size. This behavior is effectively translated in terms of the mechanical reinforcement, and it is not surprising that the effect is highly visible in the range where the nanoparticle volume is relatively low. This could also be caused by the chemical and physical properties of the matrix polymer. Sternstein and $\mathrm{Zhu}^{45}$ have showed evidences demonstrating that changes in the matrix properties close to the filler surfaces (namely, an increased density of trapped entanglements) gives rise a high mechanical reinforcement, indicating that the degree of nonlinearity are found to be highest for the lowest molecular weight polymer matrices. This statement is well-matched with the results presented in Figure 3(b). It shows that the highest reinforcement will take place for the PVA matrix which have lowest molecular weight and highest $\alpha$-parameter. The high values of $\alpha$-parameter presented in Figure 3(a) could be interpreted as the increase of trapped entanglements around the filler strongly enhancing chain reconfiguration, resulting in very high reinforcement. This explanation is also well-compatible with the prediction of the presence of glassy interphase layers around the nanoparticles, which can significantly increase the stiffness of the system. ${ }^{49}$ It seems that high thickness of the glassy layer surface around the filler will highly modify $E_{r}$

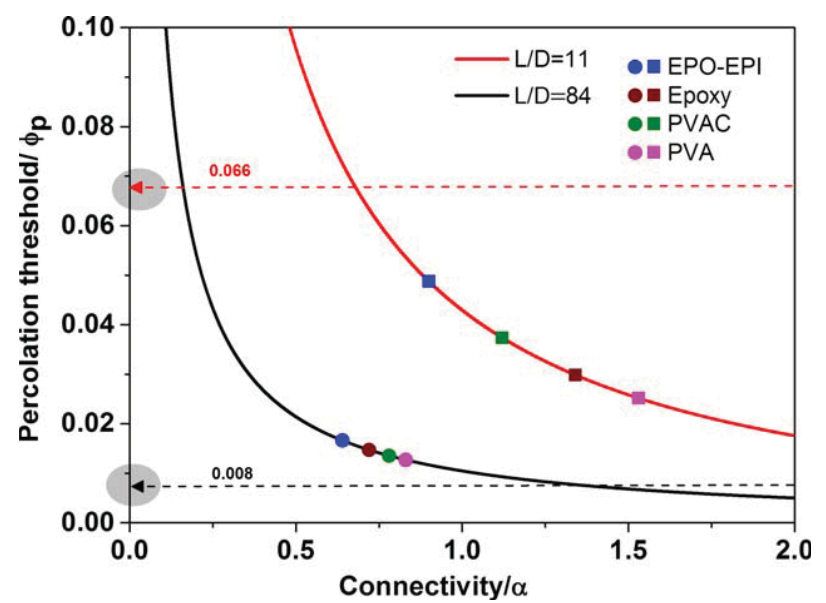

Figure 4. Percolation threshold as a function of the surface-to-surface distance criterion for connectivity for CNCs derived from tunicates ( $\mathrm{tCNCs}$, $L / D=84$ ) and CNCs derived from cotton (cCNCs, $L / D=11$ ). [Color figure can be viewed at wileyonlinelibrary.com] 
values. In addition, this could also be associated with the chemical nature of CNCs and hydrogen bonding ability with polymer matrix; PVA being the most hydrogen bonding polymer shows highest $\alpha$-parameter.

The sensibility of $\alpha$-parameter with the change in the polymer matrix can also be illustrated in a correlated manner with the percolation volume fraction values as shown in Figure 4. The lines in Figure 4, tCNCs (black solid line) or cCNCs (red solid line) show the percolation threshold from Schilling's approach. The points denote the previously determined $\alpha$ parameters extracted from Figure 2. The dotted horizontal line (black for tCNCs and red for cCNCs) represents the percolation threshold values predicted by the conventional approach. The circles and squares are added as points in the filled lines to show the percolation threshold difference for the determined connectivity parameters for both tCNCs and cCNCs. This clearly shows that the smaller is the aspect ratio of nanorods (cCNCs), the larger will be the inter-particle connectivity ${ }^{49}$ and the larger the percolation threshold discrepancy between our methods in comparison with the conventional approach. Hence, this clearly suggests that, to perform a realistic evaluation of the mechanical reinforcement in polymer nanocomposites with nanorods, the interparticle connectivity parameter should be taken into account, and it depends on the nature of the polymer.

\section{CONCLUSIONS}

In summary, in this communication we have adapted the Ouali-Takayanagi et al. model with the recently developed approach (Schilling et al.) to re-interpret the mechanical reinforcement in $\mathrm{CNC}$ reinforced polymer nanocomposites. We have shown that, depending on the nature of the polymer type and the aspect ratio of nanofiller used, the percolation threshold of the polymer nanocomposite system can change dramatically. By using experimental data for different polymer/CNC nanocomposites, we show that the smaller is the aspect ratio of the nanorods, the larger is the connectivity parameter and the lower is the percolation threshold, allowing for a more precise and realistic evaluation of the mechanical reinforcement. Our approach shows better fit with the experimental data, specifically for nanorods with lower aspect ratio. This would open a new discussion for analyzing the mechanical reinforcement of nanocomposites.

\section{ACKNOWLEDGMENTS}

The work has been financially supported by the Swiss National Science Foundation, with grant number PP00P2_159258, and by the National competence center for Bioinspired materials (NCCR). The authors express their gratitude to Christoph Weder of the Adolphe Merkle Institute, Switzerland, for sharing the results of experimental measurements and for numerous helpful discussions. JCMG would also like to acknowledge the support of Adolphe Merkle Institute for providing him the ideal facilities and conditions to carry out his scientific contributions to this project.

\section{REFERENCES}

1. Sapkota, J.; Shirole, A.; Foster, E. J.; Martinez Garcia, J. C.; Lattuada, M.; Weder, C. Polymer 2017, 110, 284.

2. Sapkota, J.; Jorfi, M.; Weder, C.; Foster, E. J. Macromol. Rapid Commun. 2014, 35, 1747.

3. Sapkota, J.; Kumar, S.; Weder, C.; Foster, E. J. Macromol. Mater. Eng. 2015, 300, 562.

4. Nicharat, A.; Sapkota, J.; Weder, C.; Foster, E. J. J. Appl. Polym. Sci. 2015, 132, DOI: 10.1002/app.42752.

5. Sapkota, J.; Natterodt, J. C.; Shirole, A.; Foster, E. J.; Weder, C. Macromol. Mater. Eng. 2017, 302, 1600300.

6. Bandera, D.; Sapkota, J.; Josset, S.; Weder, C.; Tingaut, P.; Gao, X.; Foster, E. J.; Zimmermann, T. React. Funct. Polym. 2014, 85, 134.

7. Mittal, V. Polymer Nanotubes Nanocomposites: Synthesis, Properties and Applications; John Wiley \& Sons, Inc.: Hoboken, NK, 2014.

8. Mohanty, S.; Nayak, S. K.; Kaith, B.; Kalia, S. Polymer Nanocomposites Based on Inorganic and Organic Nanomaterials; John Wiley \& Sons, Inc.: Hoboken, NK, 2015.

9. Foygel, M.; Morris, R. D.; Anez, D.; French, S.; Sobolev, V. L. Phys. Rev. B 2005, 71, 104201.

10. Nigro, B.; Grimaldi, C.; Ryser, P.; Chatterjee, A. P.; van der Schoot, P. Phys. Rev. Lett. 2013, 110, 015701.

11. Balberg, I.; Anderson, C. H.; Alexander, S.; Wagner, N. Phys. Rev. B 1984, 30, 3933.

12. Otten, R. H. J.; van der Schoot, P. Phys. Rev. Lett. 2009, 103, 225704.

13. Otten, R. H. J.; van der Schoot, P. J. Chem. Phys. 2011, 134, 094902.

14. Leung, K.; Chandler, D. J. Stat. Phys. 1991, 63, 837.

15. Chatterjee, A. P. J. Chem. Phys. 2000, 113, 9310.

16. Chatterjee, A. P. J. Phys.: Condens. Mater. 2015, 27, 375302.

17. Annamalai, P. K.; Dagnon, K. L.; Monemian, S.; Foster, E. J.; Rowan, S. J.; Weder, C. ACS Appl. Mater. Interfaces 2014, 6, 967.

18. Capadona, J. R.; Shanmuganathan, K.; Trittschuh, S.; Seidel, S.; Rowan, S. J.; Weder, C. Biomacromolecules 2009, 10, 712.

19. Capadona, J. R.; Shanmuganathan, K.; Tyler, D. J.; Rowan, S. J.; Weder, C. Science 2008, 319, 1370.

20. Capadona, J. R.; Van Den Berg, O.; Capadona, L. A.; Schroeter, M.; Rowan, S. J.; Tyler, D. J.; Weder, C. Nat. Nanotechnol. 2007, 2, 765.

21. Dagnon, K. L.; Shanmuganathan, K.; Weder, C.; Rowan, S. J. Macromolecules 2012, 45, 4707.

22. Jorfi, M.; Roberts, M. N.; Foster, E. J.; Weder, C. ACS Appl. Mater. Interfaces 2013, 5, 1517.

23. Mendez, J.; Annamalai, P. K.; Eichhorn, S. J.; Rusli, R.; Rowan, S. J.; Foster, E. J.; Weder, C. Macromolecules 2011, 44, 6827.

24. Shanmuganathan, K.; Capadona, J. R.; Rowan, S. J.; Weder, C. J. Mater. Chem. 2010, 20, 180.

25. Ouali, N.; Cavaillé, J. Y.; Pérez, J. Plast. Rubber Compos. 1991, 16, 55. 
26. Takayanagi, M.; Uemura, S.; Minami, S. J. Polym. Sci. Part C: Polym. Lett. 1964, 5, 113.

27. Stauffer, D.; Aharony, A. Introduction to Percolation Theory; Taylor \& Francis: London, 1994.

28. De Gennes, P.-G. Scaling Concepts in Polymer Physics; Cornell University Press: London, 1979.

29. Kumar, V.; Rawal, A. Polymer 2016, 97, 295.

30. Jiang, Z.; Zhang, H.; Han, J.; Liu, Z.; Liu, Y.; Tang, L. Compos. Part A: Appl. Sci. Manuf. 2016, 86, 49.

31. Pal, G.; Kumar, S. Mater. Des. 2016, 89, 129.

32. Gu, H.; Wang, J.; Yu, C. Adv. Mater. 2016, 5, 1.

33. Wang, X.; Chatterjee, A. P. J. Chem. Phys. 2003, 118, 10787.

34. White, S. I.; Mutiso, R. M.; Vora, P. M.; Jahnke, D.; Hsu, S.; Kikkawa, J. M.; Li, J.; Fischer, J. E.; Winey, K. I. Adv. Funct. Mater. 2010, 20, 2709.

35. Mutiso, R. M.; Winey, K. I. Prog. Polym. Sci. 2015, 40, 63.

36. Du, F.; Scogna, R. C.; Zhou, W.; Brand, S.; Fischer, J. E.; Winey, K. I. Macromolecules 2004, 37, 9048.

37. Mutiso, R. M.; Sherrott, M. C.; Li, J.; Winey, K. I. Phys. Rev. B 2012, 86, 214306.

38. Schilling, T.; van der Schoot, P.; Miller, M.; Dixit, M.; Meyer, H. In Percolation in Collidal Model Systems,
Proceedings of the XXVII IUPAP Conference on Computational Physics, Guwahati, India, Dec 2-5, 2015.

39. Schilling, T.; Miller, M. A.; van der Schoot, P. Europhys. Lett. 2015, 111, 56004.

40. Robertson, C. G.; Lin, C.; Rackaitis, M.; Roland, C. Macromolecules 2008, 41, 2727.

41. Sen, S.; Thomin, J. D.; Kumar, S. K.; Keblinski, P. Macromolecules 2007, 40, 4059.

42. Zhao, D.; Ge, S.; Senses, E.; Akcora, P.; Jestin, J.; Kumar, S. K. Macromolecules 2015, 48, 5433.

43. Davris, T.; Mermet-Guyennet, M. R.; Bonn, D.; Lyulin, A. V. Macromolecules 2016, 49, 7077.

44. Berriot, J.; Montes, H.; Lequeux, F.; Long, D.; Sotta, P. Macromolecules 2002, 35, 9756.

45. Sternstein, S. S.; Zhu, A.-J. Macromolecules 2002, 35, 7262.

46. Gavrilov, A. A.; Chertovich, A. V.; Khalatur, P. G.; Khokhlov, A. R. Macromolecules 2014, 47, 5400.

47. Tang, L.; Weder, C. ACS Appl. Mater. Interfaces 2010, 2, 1073.

48. Thorkelsson, K.; Bronstein, N.; Xu, T. Macromolecules 2016, 49, 6669.

49. Pakzad, A.; Simonsen, J.; Yassar, R. S. Compos. Sci. Technol. 2012, 72, 314. 